\title{
DELAY IMPROVEMENT USING EFFICIENT HARQ-SCHEDULER IN LTE
}

\author{
Srinivasa R.K \\ Research Scholar, Department of Electronics \& Communication Engineering, \\ BIT, Visvesvaraya Technological University, Bangalore, India. \\ Hemanth Kumar A.R \\ Professor and Principal Investigator (R\&D) \\ Department of Electronics \& Communication Engineering, BIT, Bangalore, India
}

\begin{abstract}
Long Term Evolution (LTE) is standardised by the 3GPP to provide a high data rate up to $100 \mathrm{Mbps} / 50 \mathrm{Mbps}$ in Rel8 for downlink/uplink transmission respectively and effectively increases with the addition of new features including MIMO, CA. LTE effectively can operate with scalable bandwidths ranging from $1.4 \mathrm{MHz}$ up to $20 \mathrm{MHz}$ with varied Resource blocks ranging from 6 to 100. Resource allocation is a key role in order to achieve the goals of the Long Term Evolution (LTE) and scheduling mechanisms incorporated in the MAC Layer of the architecture contribute to this goal. Scheduling is the process in which the resources are allocated in both Time and Frequency domain to users. In this paper, evolved scheduling algorithm is proposed for reducing the delay for the application of critically without compromising the cell throughput and fairness in comparison with Round Robin, Best-CQI schedulers.
\end{abstract}

Key words: LTE, Scheduler, MAC, Round Robin, Best-CQI.

Cite this Article: Srinivasa. R.K and Hemanth Kumar. A.R, Delay Improvement using Efficient HARQ-Scheduler in LTE. International Journal of Electrical Engineering and Technology, 11(5), 2020, pp. 120-126.

http://iaeme.com/Home/issue/IJEET?Volume=11\&Issue=5

\section{INTRODUCTION}

With the increase in the demand of the High speed data for various real time / non-real time applications Long Term Evolution (LTE) is standardised by the 3GPP in Release 8, as the successor of the Universal Mobile Telecommunication System (UMTS/WCDMA) as new air interface technology.

The radio access technology chosen for LTE system is the Orthogonal Frequency Division Multiple Access (OFDMA), in both Time Division Duplexing (TDD) and Frequency Division Duplexing (FDD), because his robustness to the selectivity of multi path channels [1]. 
The high peak transmission rate reaches the LTE system is 100 Mbps in downlink (DL) and $50 \mathrm{Mbps}$ in uplink (UL) for Category 3 UE. LTE Rel8/9 defines five different UE categories, detailed description of terminals are defined in 3GPP technical doc UE Radio Access Capabilities TS 36.606 as per table below.

Table 1 UE categories and Peak DL \& UL Data Rates

\begin{tabular}{|c|c|c|c|c|c|c|c|c|}
\hline & \multicolumn{8}{|c|}{ UE Category } \\
\hline & 1 & 2 & 3 & 4 & 5 & 6 & 7 & 8 \\
\hline $\begin{array}{l}\text { Downlink Peak Data Rate } \\
(\mathrm{Mbit} / \mathrm{s})\end{array}$ & 10 & 50 & 100 & 150 & 300 & 300 & 300 & 3000 \\
\hline $\begin{array}{l}\text { Uplink Peak Data Rate } \\
(\mathrm{Mbit} / \mathrm{s})\end{array}$ & 5 & 25 & 50 & 50 & 75 & 50 & 150 & 1500 \\
\hline Maximum MCS & \multicolumn{8}{|c|}{ 64QAM } \\
\hline Maximum uplink MCS & \multicolumn{4}{|c|}{ 16QAM } & 64QAM & 16QAM & \multicolumn{2}{|c|}{ 64QAM } \\
\hline $\begin{array}{l}\text { Max. number of layers for } \\
\text { downlink spatial Multiplexing }\end{array}$ & 1 & & 2 & & 4 & Signal & separ & ely \\
\hline
\end{tabular}

LTE To achieve the performance objectives, LTE employs the several techniques such as MIMO[2], Diversity, CA, HARQ

This technology presents very interesting use cases of multi users with varied QOS, latency/delay needs depending on the type of application as end user being used. There is a need to present an evolved scheduler which can provide less delay comparatively by allocating the resources with better fairness and need.

The Scheduler implementation is vendor dependent and 3GPP has no role to play in it. This paper presents an evolved scheduling algorithm for downlink transmission in LTE cellular network. The performances of the proposed evolved scheduler are evaluated and compared to the basic Round Robin and Best-CQI scheduler. The proposed algorithm is compared for the latency/delay. Subsequent paper is organised as follow: In Section 2, LTE downlink scheduling mechanism are discussed. In Section 3, Evolved scheduler flow is discussed, followed basic algorithms and Test use case/Scenario, Numerical results in Section 4, 5 followed by Conclusion in Section 6 .

\section{DOWNLINK SCHEDULERS IN LTE}

MAC Layer of LTE has scheduler which allocates the resources in both Frequency and Time domain for all the active UEs. The Resources are allocated for both Downlink and Uplink transmission using the Downlink Control Information (DCI) using the PDCCH channel on the Downlink. In LTE the resources are allocated at every TTI duration. TTI duration in LTE is $1 \mathrm{msec}$ with each Frame divided in to $10 \mathrm{msec}$ having 10 SubFrames and each SubFrame have 2 slots of each having 0.5 msec.

Depending on the system bandwidth the Resource blocks aka Physical Resource Blocks (PRB) in LTE is consecutive 12 subcarriers each of $15 \mathrm{KHz}$ in frequency domain [180 KHz] and 7 or 6 OFDMA symbols in the time domain of two slot of $0.5 \mathrm{msec}$. Minimum allocation unit in downlink LTE is called Physical Resource Block (PRB) which consists of two RBs where each PRB is assigned to a UE in $1 \mathrm{~ms}$ time duration

Resource allocation to UE in the Downlink is via calculated SINR which is computed in UE and CQI, found through CQI mapping process from SINR, is sent within uplink channel to eNB. The value of CQI corresponds to MCS for a specific Block Error Rate (BLER)[3]. The BLER should not be more than a threshold. The recommended threshold is $10 \%$ for LTE 
[4]. CQI to SNR mapping with different multiple antenna techniques and HARQ retransmissions can provide a reliable estimate [5]

Many applications are sensitive to delay depending the QOS needs or based on GBR / Non-GBR applications. Its needed to fairly handle the real time applications which are having the multiple retransmissions due to repeated NACKs due to more BLER or not able to decode the Downlink data packets.

\subsection{Round Robin Scheduler}

The RR scheduler schedules UEs in a round robin manner by allocating equal resource share. It provides parity, however the disadvantage of this approach is that UEs with sub-optimal CQIs may be allocated Physical Radio Resources (PRBs) that may go un-utilised, thus reducing the overall cell throughput.

\subsection{Best-CQI Scheduler}

Best-CQI scheduler selects the Schedulable UEs based on the reported CQI in the Uplink based on the Downlink SINR experienced by UE. The UEs with the highest CQI become candidates for scheduling at eNodeB thereby increasing the overall cell throughput. The disadvantage of this approach is that UEs with lower CQI are not fairly scheduled or denied scheduling at some time, thus being starved for throughput and leading to degraded user experience.

\subsection{Proportional Fair Scheduler}

PF schedulers provides a balance by maintaining the fairness as well as allocation of Resources to the UEs with Best-CQI there by maximising the cell throughput. Disadvantage of this approach is complex implementation and also doesn't consider the delay increases as an input while scheduling the UEs.

The fairness and the cell throughput is maintained by considering the instantaneous Data Rate requested by the $\mathrm{k}^{\text {th }}$ UE at the current TTI cycle among the $\mathrm{n}$ UEs in the cell over the average throughput $\left(\mathrm{A}_{\text {tput }}\right)$ over the previous window length of tc.

$$
\text { PF Scheduler }=D_{\text {req }} k, n(t) / A_{\text {tput }} k, n(t)
$$

\section{PROPOSED EVOLVED SCHEDULER}

Evolved scheduler is here on called as "HARQ-Scheduler" which takes in to the account of the UEs having more retransmissions due to NACKs. Many studies have shown that increased in number of retransmissions will neither help increasing the cell throughput nor the fairness. HARQ-Scheduler prioritise the UEs/application with more than one retransmission based on the Redundant Version (RV) with equal fairness as that of the UEs with higher CQI reported. The approach is followed by reducing the MCS at the scheduler by the eNodeB based on the current CQI \& MCS being used in the Downlink [6] there by adding more redundant bits which helps the protected message to from corruption making UE to decode it without any errors. Multiple proposals for the MCS-CQI mapping has been done in the green field LTE [7]

\subsection{HARQ-Scheduler Flow chart}

Figure 1. below shows the high level flow of the proposed evolved HARQ-Scheduler where the first Subframe is prioritised for the UEs with High CQI and the next Subframe is prioritised for the UEs having high retransmissions followed by the Higher CQI thereby improving the delay of the UEs undergoing more retransmissions. 


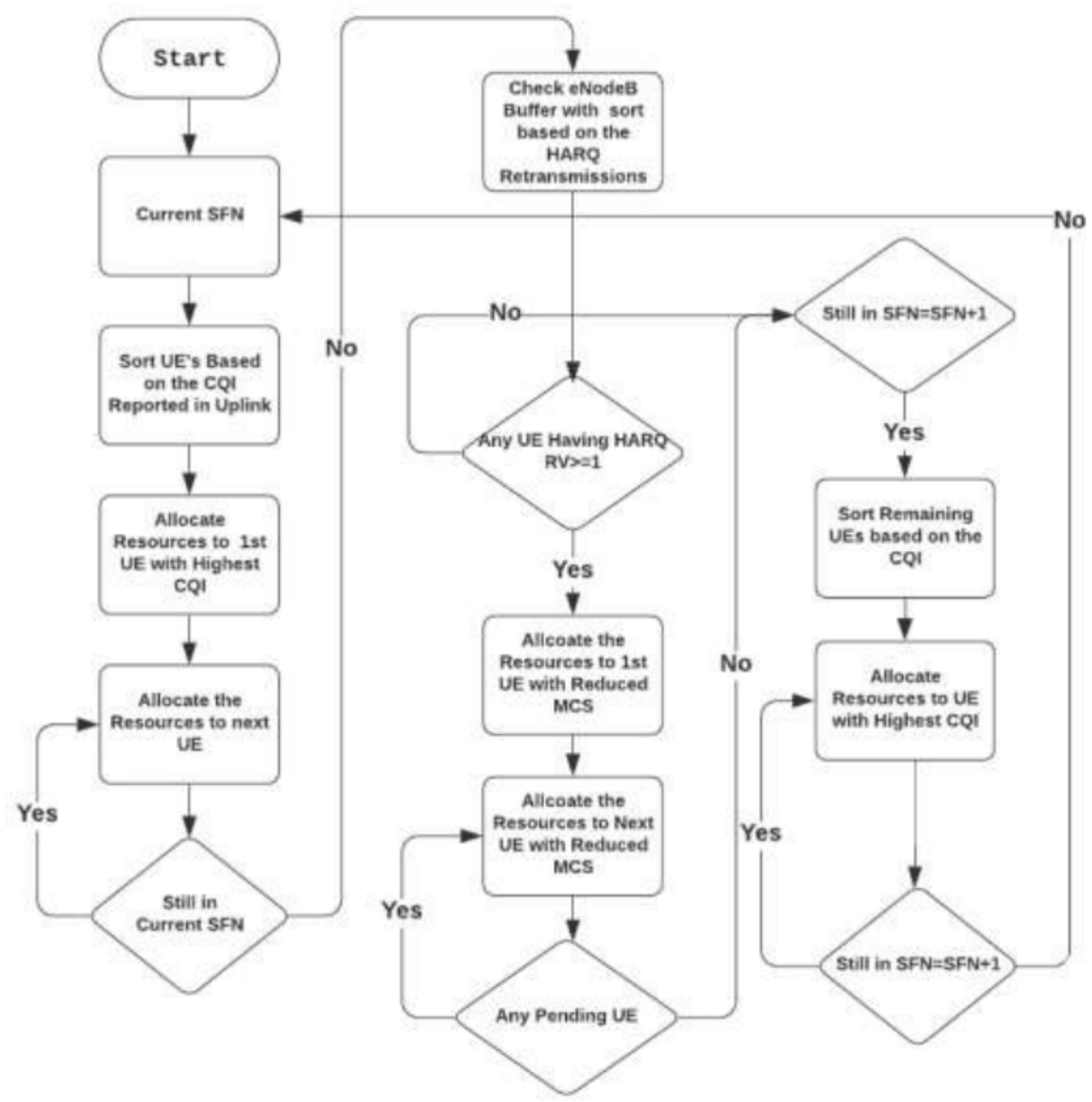

Figure 1 Flow Chart : Evolved HARQ-Scheduler

Flow chart also emphasis on the Modulation and Coding Scheme (MCS) reduction based on the CQI reported for the UEs with more retransmissions as detailed in algorithm under section 3.2

\subsection{Algorithm : UEs Prioritisation and CQI/MCS adoption in Downlink for the UEs with $R V>=1$}

1. Prioritise the UEs having RV $>=1$ in SFN 2, 4, 6, 8 and so on

2. Any UE with $R V>=1$ in the eNode B Buffer waiting for the Data Retransmission

3. Check the CQI/MCS being severed based on the CQI reported

4. Adopt the CQI/MCS according to the below logic

If CQI being reporter is in the range CQI10-15 adopt to CQI-9

Else If

CQI being reporter is in the range CQI7-9 adopt to CQI-6

Else If 
CQI being reporter is in the range CQI2-6 adopt to CQI-1

Else

Use the reported CQI.

5. Map the MCS and TBS as per the specifications according the reduced CQI table.

\section{TEST SCENARIO AND USE CASE}

LTE effectively can operate with scalable bandwidths ranging from $1.4 \mathrm{MHz}$ up to $20 \mathrm{MHz}$ with varied Resource blocks ranging from 6 to 100. For the purpose of explanation let us consider the cell deployed with $1.4 \mathrm{MHz}$ having 6 PRBs and with 3 UEs at Near Cell, Mid Cell and Edge cell as shown below Figure 2.

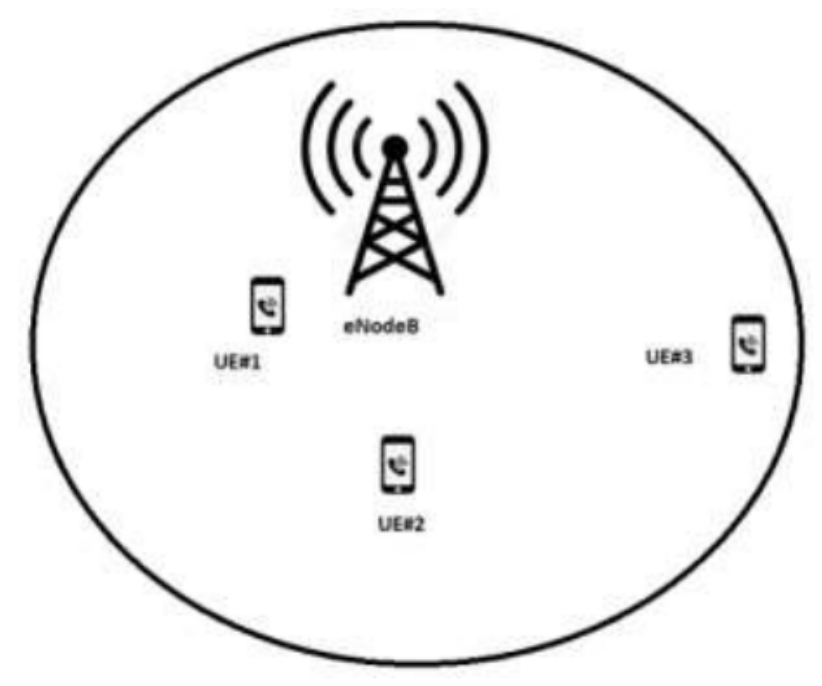

Figure 2 Base station with 3UEs Near Cell(UE\#1), Mid Cell(UE\#2) and Edge Cell(UE\#3)

UE\#1 : Near cell reports very high CQI compared to UE\#2 and UE\#3.

UE\#3 : Will be either be denied from Resource allocation or experience higher retransmission being at edge cell due to the decoding issues or higher BLER.

Below is the view of PRB allocation for the UEs across different TTI cycles.

TTI1 : UE1, UE1, UE2, UE2, UE1, UE3

TTI2 : UE3, UE1, UE2, UE3, UE1, UE2

The same has been illustrated in the below table Modified PRB allocation Map for 1.4MHz BW with 3 Users

Table 2 Modified PRB allocation Map for 1.4MHz BW with 3 Users

\begin{tabular}{|c|c|c|}
\hline RB's-1.4MHz & SFN\#1 & SFN\#2 \\
\hline PRB1 & UE1 & UE3 \\
\hline PRB2 & UE1 & UE1 \\
\hline PRB3 & UE2 & UE2 \\
\hline PRB4 & UE2 & UE3 \\
\hline PRB5 & UE1 & UE1 \\
\hline PRB6 & UE3 & UE3 \\
\hline
\end{tabular}




\section{SIMULATION PARAMETERS AND RESULTS}

\subsection{Simulation Parameters}

In this section, we will simulate and discuss the performance of the proposed HARQScheduler and compare the obtained result with performances of basic scheduling algorithms, such as Round Robin, Best-CQI scheduler for downlink transmission in LTE system. The simulations are carried out for frequency-selective channels mode-led by ITU for PedestrianB (Ped-B) channels. The simulations are performed for a time length equivalent of 1000 subframes for users ranging from 5 to 25 , choosing the bandwidth of $1.4 \mathrm{MHz}$ containing $6 \mathrm{RBs}$ and 72 occupied sub-carriers

\subsection{Simulation Results}

Based on the simulation results its evident the delay is consistently less in proposed HARQScheduler compared to the Round Robin and Best CQI. The experiment is simulated for the various base station transmit power ranging from 0 to $45 \mathrm{dBm}$ as shows in Fig 3 .

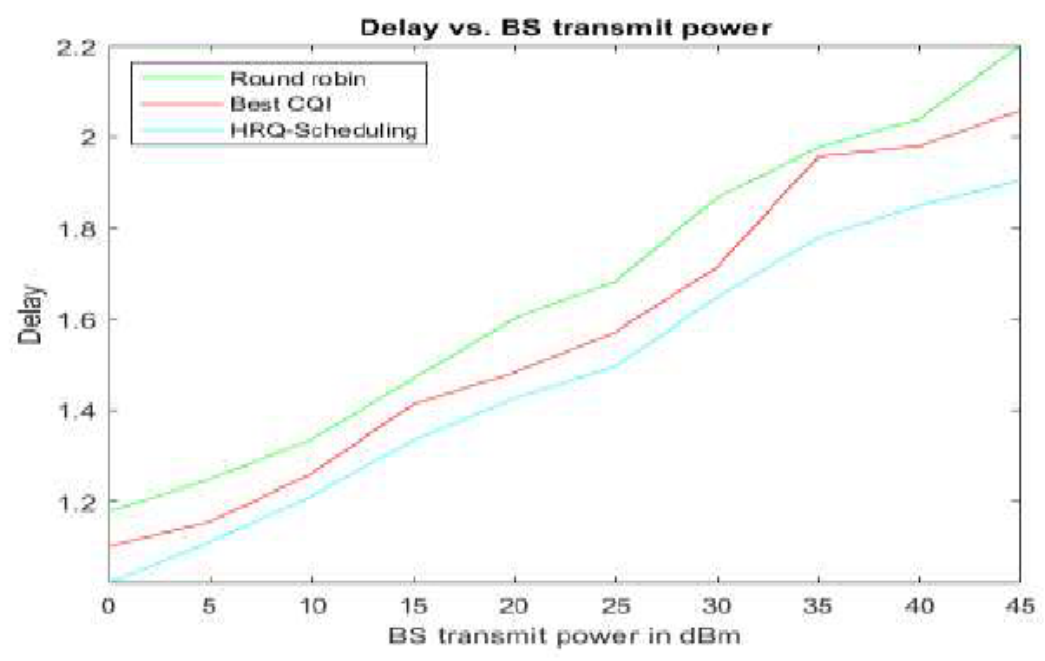

Figure 3 Delay Vs Base Station Transmit Power

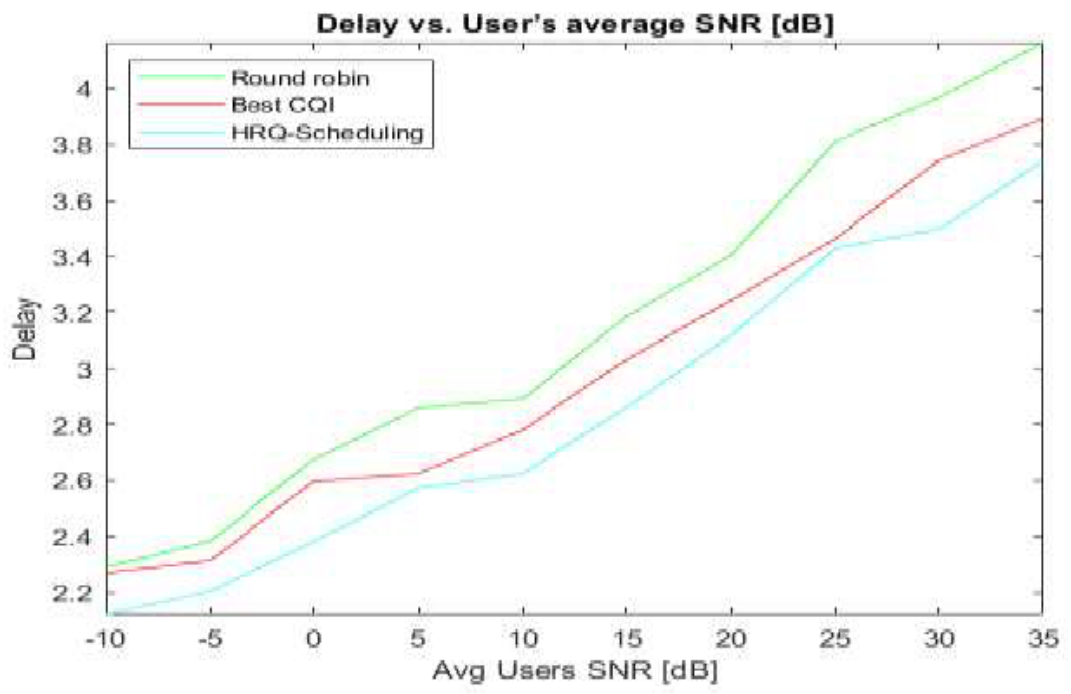

Figure 4 Delay Vs Average User's SNR

Also, the fairness in the system in the proposed HARQ-Scheduler is seen better compared to the traditional Round Robin and Best CQI. The proposed HARQ-Scheduler exploit the 
efficient use of the Physical Resource Block allocation based on the applications as well which provides an advantage of improvement in fairness with reduced delay as should in Fig 5 .

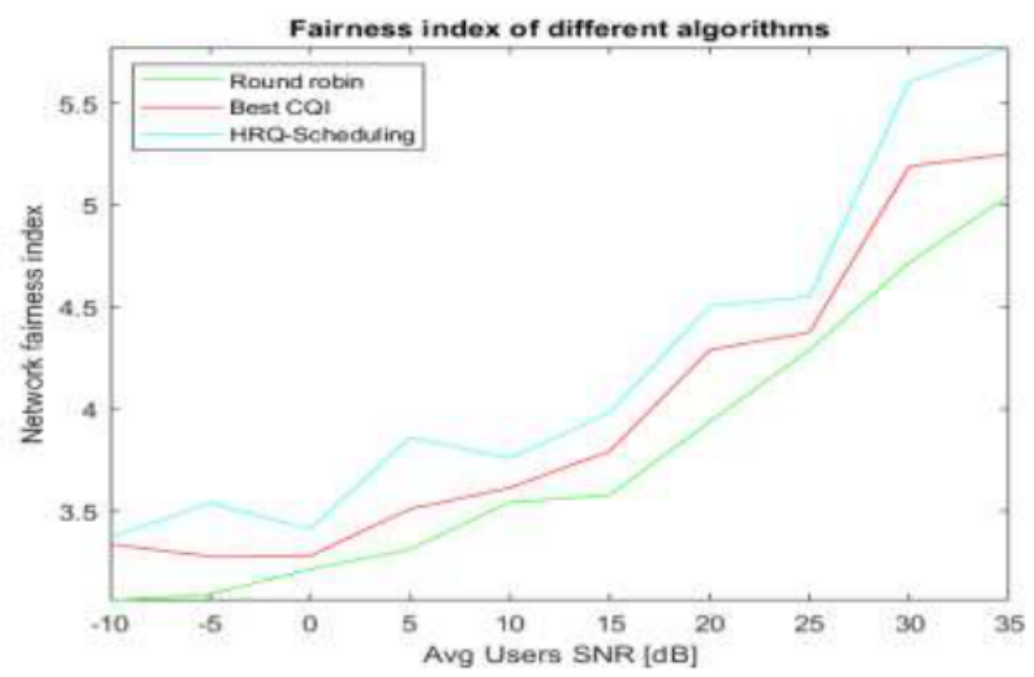

Figure 5 Fairness Index of Different Algorithms Vs Average User's SNR

\section{CONCLUSION}

The main focus of this paper is to evaluate a comprehensive study on various LTE scheduling schemes. We have proposed and evolved HARQ-Scheduler scheme for the downlink transmission mode in LTE and its performance wrt Delay is compared with the other two existing scheduling schemes, namely Best-CQI and RR schedulers. The results from simulations show that the proposed evolved HARQ-Scheduler performs the best as compared to the other two schedulers especially in terms of Delay and Fairness. This is mainly achieved by the way of the Physical Resource Blocks(PRB) allocations in every Transmission Time Internal(TTI) or SubFrame(SFN). Besides that, it is also interesting to study the system throughput and spectral efficiency performance for all the schedulers in our further studies.

\section{REFERENCES}

[1] 3GPP TS 36.212 Version 10.0.0 Release 10, Evolved Universal Terrestrial Radio Access (E-UTRA): Multiplexing and channel coding, March, 2009

[2] Technical White paper: iLong Term Evolution (LTE): A Technical Overview,î by Motorola.

[3] Raymond H. Y. Louie, "Open-Loop Spatial Multiplexing and Diversity Communications in Ad Hoc Networks", Revised version. Submitted to IEEE Tr. Info Theory, 17 september 2010.

[4] C. Mehlf,hrer, M. Wrulich, J. C. Ikuno, D. Bosanska, and M. Rupp, "Simulating the long term evolution physical layer," in Proc. of the 17th European Signal Processing Conference (EUSIPCO 2009), Glasgow, Scotland, 2009, p. 124.

[5] Downlink SNR to CQI Mapping for Different Multiple Antenna Techniques in LTE, International Journal of Information and Electronics Engineering, Vol. 2, No. 5, September 2012

[6] 3GPP TS 36.213 version 12.3.0 Release 12 Evolved Universal Terrestrial Radio Access (E-UTRA); Physical layer procedures Table 7.2.3-1: 4-bit CQI Table

[7] CQI-MCS Mapping for Green LTE Downlink Transmission, Proceedings of the AsiaPacific Advanced Network 2013 v. 36, p. 74-82.ISSN 2227-3026 